10-1-2015

\title{
Use of High-Speed X ray and Video to Analyze Distal Radius Fracture Pathomechanics.
}

\author{
Christina Gutowski \\ Thomas Jefferson University \\ Kurosh Darvish \\ Temple University \\ Frederic E. Liss \\ Rothman Institute, Thomas Jefferson University
}

Asif M. Ilyas

Rothman Institute, Thomas Jefferson University

Christopher M. Jones

Rothman Institute Thomas Jefferson University

Follow this and additional works at: https://jdc.jefferson.edu/orthofp

Part of the Orthopedics Commons

Let us know how access to this document benefits you

\section{Recommended Citation}

Gutowski, Christina; Darvish, Kurosh; Liss, Frederic E.; Ilyas, Asif M.; and Jones, Christopher M., "Use of High-Speed X ray and Video to Analyze Distal Radius Fracture Pathomechanics." (2015). Department of Orthopaedic Surgery Faculty Papers. Paper 80.

https://jdc.jefferson.edu/orthofp/80

This Article is brought to you for free and open access by the Jefferson Digital Commons. The Jefferson Digital Commons is a service of Thomas Jefferson University's Center for Teaching and Learning (CTL). The Commons is a showcase for Jefferson books and journals, peer-reviewed scholarly publications, unique historical collections from the University archives, and teaching tools. The Jefferson Digital Commons allows researchers and interested readers anywhere in the world to learn about and keep up to date with Jefferson scholarship. This article has been accepted for inclusion in Department of Orthopaedic Surgery Faculty Papers by an authorized administrator of the Jefferson Digital Commons. For more information, please contact: JeffersonDigitalCommons@jefferson.edu. 


\section{Titles:}

Use of High-Speed Xray and Video to Analyze Distal Radius Fracture Pathomechanics

\section{Author information \& Order:}

\section{(1) Christina Gutowski, MD MPH}

Department of Orthopaedic Surgery, Thomas Jefferson University

1025 Walnut St. Room 516 College Building

Philadelphia, PA 19107

gutowski1@gmail.com

(2) Kurosh Darvish, PhD

Department of Mechanical Engineering, Temple University

1947 N. $12^{\text {th }}$ Street

Philadelphia, PA 19122

kdarvish@temple.edu

(3) Frederic E. Liss, MD

Rothman Institute, Thomas Jefferson University

925 Chestnut St

Philadelphia, PA 19107

fred.liss@rothmaninstitute.com

(4) Asif M. Ilyas, MD

Rothman Institute, Thomas Jefferson University

925 Chestnut St

Philadelphia, PA 19107

asif.ilyas@rothmaninstitute.com

(5) Christopher Jones, MD (*Corresponding Author)

Rothman Institute, Thomas Jefferson University

925 Chestnut St

Philadelphia, PA 19107

Phone: 800-321-9999; fax: 215-503-0530

christopher.jones@rothmaninstitute.com

Keywords: distal radius fracture; high-speed xray; video xray; fracture biomechanics; wrist guard

Disclosure: None of the authors of this manuscript have any conflicts of interest or relationships to disclose. Nothing of benefit was received by any of the authors in the production of this manuscript. 


\section{SYNOPSIS:}

Distal radius fractures are among the most common fractures treated by orthopaedists, and result in significant morbidity and cost across all age groups of the population. In other anatomic locations such as the cervical spine, the pathomechanics of fracture development are well-described, as result of in vivo and in vitro laboratory experiments and finite element modeling. The purpose of this study is to investigate the failure sequence of the distal radius during a simulated fall onto an outstretched hand, utilizing cadaver forearms and high-speed x-ray and video systems. This apparatus will record the beginning and propagation of bony failure, ultimately resulting in distal radius or forearm fracture. The effects of three different wrist guard designs will also be investigated using this system. Serving as a proof of concept analysis, this study supports this imaging technique to be utilized in larger studies of orthopaedic trauma and protective devices, and specifically for distal radius fractures. 


\section{KEY CONCEPTS:}

(1) Distal radius fractures are common injuries, especially in the pediatric and elderly populations, that account for significant morbidity and mortality

(2) The combination of high-speed x-ray and video imaging has been utilized in other areas of medicine to investigate physiologic movement, joint kinematics, and fracture propagation. This type of recording system has not been utilized in the study of distal radius fracture pathomechanics.

(3) This imaging technique proved feasible with a drop tower apparatus consisting of a potted cadaver forearm, weights dropped to simulate a fall onto an outstretched hand, two identical video recorders, an x-ray source, and an image intensifier.

(4) In the presented pilot study, when impacted, the distal radius was found to shift in a volar direction with respect to the proximal carpal row, with the carpus also hyperextending. In elderly wrists, failure of the distal radius began as compressive failure on the dorsal cortex followed by tensile failure of the volar cortex. In younger wrists, both cortices tended to fail simultaneously.

(5) As a proof of concept, this study confirms that future studies with larger cadaveric samples can be analyzed with high-speed xray and video to better understand distal radius fracture pathomechanics. 


\section{INTRODUCTION}

Distal radius fractures are among the most common injuries treated by orthopaedists. ${ }^{1}$ and the most frequently-diagnosed fracture in women. ${ }^{2}$ In fact, healthy females 60 years of age have a 17\% chance of sustaining a fracture of the distal radius. ${ }^{3}$ The incidence of this injury has increased significantly over the last several decades, a trend noted both domestically and internationally. 4,5,6 Distal radius fractures result in considerable pain and loss of productivity, often require surgical treatment, and are an indicator of increased risk of other fractures. ${ }^{7}$ A 2011 report estimated that $\$ 170$ million were paid by Medicare in 2007 towards treatment of distal radius fractures, and the future burden to Medicare will approach $\$ 240$ million if trends in utilization of internal fixation continue as anticipated. ${ }^{8}$ These fractures are also a common injury in the pediatric population, ${ }^{9}$ accounting for $25 \%$ of all fractures in this age group ${ }^{10}$. The estimated cost associated with treating pediatric distal radius fractures is upwards of $\$ 2$ billion per year in the US. ${ }^{11}$ Given the high incidence of distal radius fractures in young and old alike, and the significance as both a personal injury and a public health concern, further understanding of the biomechanical mechanism of this fracture is essential. Moreover, improving our understanding of failure mechanisms of the distal radius might enable us to develop both improved treatments and preventative or protective devices.

The failure sequence of the distal radius during a typical fall on an outstretched hand is not well-described in the literature. Ulrich et al (1999) utilized high-resolution images captured by a micro-CT scanner and micro-finite element analysis techniques to calculate stress and strain on areas of the distal radius at the tissue-level, and quantify load transfer through the trabecular network. ${ }^{12}$ However, this in vivo study did not simulate an 
actual fracture to the distal radius; only sub-failure loads were applied to the bone, through the scaphoid or lunate. In other anatomic locations, the bony sequence of injury is better understood. In the cervical spine, for example, axial loading has long been considered to be the force behind the mechanism of vertebral burst fracture. Both in vivo and in vitro laboratory experimental studies as well as finite element modeling can subsequently predict areas of the veterbral body where failure is likely to begin and propagate when exposed to a given load. ${ }^{13}$ No study exists that investigates the failure mechanism of the distal radius to this extent.

There are several published research studies that have evaluated the effectiveness of wrist guards in protecting against distal radius fractures. ${ }^{14}$ They employ a variety of test setups to simulate an impact across the wrist by using force-measuring platforms and strain gauges to calculate the force applied to the distal radius. These studies were conducted both with the wrist bare and while wearing a protective guard. Investigations using both cadavers and human subjects suggest that various forms of wrist guards can be helpful in reducing momentum and allowing for a higher impulse to be applied to the wrist/brace construct before distal radius failure,.15,16,17,18 While these biomechanical data points can provide valuable support for wrist guard utilization, they do not provide direct information regarding the biomechanics of force transmission across the wrist and forearm during a fall.

In order to better understand the sequence of events, specifically the relative motion and failure sequence that occurs during a distal radius fracture, the authors believe that high-speed imaging techniques, combined X-ray and video imaging, offer promise. These techniques have been utilized in other fields, although the literature is scarce 
regarding their use in orthopaedic trauma, especially in investigating distal radius fractures. For example, in veterinary medicine, a high-speed x-ray system was described by Snelderwaard et al to analyze the rapid projection of a small animal's tongue to capture prey during a feeding experiment. ${ }^{19}$ Techniques of imaging fluid movement ${ }^{20}$ and the motion of artificial heart valves ${ }^{21}$ have also been described by using high-speed modern video systems.

In the fields of orthopaedic sports medicine and arthroplasty, dynamic fluoroscopy has been investigated to elucidate joint kinematics in vivo in attempt to understand the weightbearing forces, shear stresses, and relative motion of the joint. 22,23 To the best of our knowledge, combined high speed X-ray and video has not been used to investigate fracture pathomechanics. The objective of the current report is to serve as a proof of concept demonstration, evaluating the feasibility of combined high speed X-ray and video imaging techniques applied to a fracture model. Specifically, we sought to investigate the fracture mechanism of the distal radius from a typical fall on outstretched hand and the effectiveness of various wrist guard designs.

\section{EXPERIMENTAL DESIGN}

Eight fresh-frozen cadaveric arms were thawed at room temperature for 24 hours. The elbow was disarticulated and all soft tissues were removed from the proximal forearm. The proximal radius and ulna were potted using R1 FastCast Urethane casting resin. The forearms were positioned vertically in an impact testing apparatus with wrist preloaded in extension [Figure 1]. Impact force, provided by a $45 \mathrm{~kg}$ drop weight, was transferred to the arm via a load transfer plate assembly that restricted motion to the vertical direction using 
guide shafts. A rigid weight stopper prevented excessive compression of the arm. Weight was dropped on bare cadaver wrists, as well as those protected by one of three wrist guard designs.

The drop test was photographed with high-speed video and x-ray using a mechanical trigger on the drop test apparatus [Figure 2]. The x-ray equipment consisted of a source radiating in the medial-lateral direction (150 kVP, Varian Medical Systems, Palo Alto, CA)and a 550mm image intensifier (VJ Technologies, Inc., Bohemia, NY) which captured dynamic lateral x-ray images of the distal forearm and hand during impact loading. This machine captured images at 1000 frames per second (fps). Two high-speed video acquisition systems (Memrecam GX-1, nac Image Technology, Simi Valley, CA) were positioned adjacent to the radiation source to record a video of the wrist set-up viewed from the lateral direction, as well as the dynamic lateral x-ray images. Images were also recorded at 1000 fps. All image data was stored in a hard drive and later analyzed with Quicktime 7 video player using the zoom feature and image processing functions to best visualize the region of interest. Images were analyzed on a frame by frame basis to characterize the fracture sequence.

Impact force was measured using 2 load cells. Displacement and acceleration of the load transfer plate was measured with a string potentiometer and accelerometer, respectively. Strain in the distal radius and ulna were measured using strain gage rosettes. These measurement devices allowed for more traditional biomechanical data to be captured, consistent with those data described by previous studies, although these are not the focus of this report. 
Three types of wrist guards were used including a prototype created by the researchers. The two commercially-available wrist guards were the Seirus Jam Master II (Seirus, San Diego, CA), incorporating both volar and dorsal protective plates meant to be worn above a ski/snowboard glove, and the Dakine volar wrist guard (Dakine, Hood River, OR), which is lower-profile and consists of an aluminum volar plate. The prototype design included a viscoelastic foam core and thin rigid shell on the volar side of a neoprene sleeve.

[Figure 3] The appropriate size guard was secured to the cadaver hand for impact tests. The high-speed x-ray images and videos were studied for patterns of relative motion and location/sequence of fracture development. Each forearm was first tested with nondestructive loads at approximately $30 \%$ of the anticipated failure load with and without the wrist guards. Destructive tests were then performed alternating between wrist guard type and no guard.

Tags: distal radius fracture simulation, wrist guard, drop tower apparatus, high-speed video x-ray

\section{EARLY RESULTS AND FUTURE DIRECTION}

A better understanding of the pathomechanics of distal radius fractures can be gleaned by studying the videos of dynamic wrist motion when exposed to axial load, resulting eventually in fracture of the distal radius. In our study of eight cadaver forearms, in both protected and unprotected wrists the common failure mechanism occurred through axial compression of the extended carpus, causing the radius to shift slightly in a volar direction relative to the proximal carpal row, and the proximal carpal row to hyperextend. [Figures 4 and 5] We measured the radiocapitate angle using a goniometer to quantify the 
proximal carpal row hyperextension. From preload to failure the capitate extended from 42 to 55 degrees on average. In several videos, the capitate appeared to abut the dorsal radial rim and possibly transfer load directly to the dorsal cortex of the radius.

Being a proof of concept analysis, our study lacked power to make definitive conclusions on sequence of failure during fracture; however, it appeared that in older cadavers, failure occurred first in the dorsal cortex followed by tension failure of the volar cortex, while in younger cadavers both cortices failed simultaneously. When studying the wrist braces, it was noted that the volar brace tended to slide proximally down the wrist, affording less impact absorbance against axial load as compared to the prototype brace, which did not migrate from its intended position. We also noted that the volar splint appeared to function by transferring a portion of the wrist extension load through the rigid support to the forearm straps, bypassing the wrist. Transferring the impact force proximally to protect the distal radius did potentially risk development of a proximal fracture, which occurred in the mid-radial shaft and at the bone-potting cement interface in two specimens. Also, analyzing the high speed video data provided other insight helpful in optimizing the guard design. For example, it was noted that the viscoelastic cushion which was designed to fully compress to absorb the maximal force, only compressed a fraction of its thickness. Video analysis could be used to evaluate the compressibility and effectiveness of other types and thicknesses of material, including layered composites, which otherwise might be challenging to estimate their material properties.

Qualitative observations made from studying the high-speed video and x-rays combined with quantitative analysis of strain gauge and load cell data, can offer valuable insight into the distinct biomechanical failure sequence that occurs in distal radius 
fractures. By understanding this to a greater extent, personal protective devices can be enhanced to optimize effectiveness. It is the authors' belief that this pilot study offers sound proof of concept and support for this imaging technique to be utilized in larger studies of orthopaedic trauma, and specifically for distal radius fractures, in the future.

Tags: pathomechanics of distal radius fracture, fracture propagation, effect of wrist guard, high-speed video x-ray

${ }^{1}$ Lidstrom A. Fractures of the distal end of the radius. A clinical and statistical study of the end results. Acta Orthop Scand.1959;41(suppl):1-118.

${ }^{2}$ Lauritzen JP, Schwarz P, Lund B et al. Changing incidence and residual lifetime risk of common osteoporosis-related fractures. Osteopor Int.1993;3:127-132.

${ }^{3}$ Lauritzen JP, Schwarz P, Lund B et al. Changing incidence and residual lifetime risk of common osteoporosis-related fractures. Osteopor Int.1993;3:127-132.

${ }^{4}$ Melton L, Amadio P, Crowson C, O'fallon W. Long-term trends in the incidence of distal forearm fractures. Osteoporosis Int.1998;8:341-348.

${ }^{5}$ Alffram PA, Bauer GC. Epidemiology of fractures of the forearm. A biomechanical investigation of bone strength. J Bone Joint Surg. 1962;44-A:105-114.

${ }^{6}$ Bengner $\mathrm{U}$, Johnell $\mathrm{O}$. Increasing incidence of forearm fractures. A comparison of epidemiologic patterns 25 years apart. Acta Orthop Scand.1985;56:158-160.

${ }^{7}$ Mallmin H, Ljunghall S, Persson I, Berstrom R. Risk Factors for Fractures of the Distal Forearm: a Population-Based Case-Control Study. Osteopor Int 1994;49:298-304.

${ }^{8}$ Shauver MJ, Yin H, Banerjee M, Chung KC. Current and future national costs to Medicare for the treatment of distal radius fracture in the elderly. J Hand Surg Am. 2011;36:12821287.

${ }^{9}$ Cooper C, Dennison EM, Leufkens HGM, Bishop N, van Staa TP. Epidemiology of childhood fractures in Britain: a sstudy using the general practice research database. J Bone Min Res.2004;19(12):1976-1981.

${ }^{10}$ Nellans KW, Kowalski E, Chung KC. The epidemiology of distal radius fractures. Hand Clin.2012;28(2):113-125.

${ }^{11}$ Ryan LM, Teach SJ, Searcy K, Singer SA, Wood R, Wright JL, Chamberlain JM. Epidemiology of pediatric forearm fractures in Washington, DC. J Trauma. 2010;69(4S):S200-205. 
12 Ulrich D, van Rietbergen B, Laib A, Ruegsegger P. Load transfer analysis of the distal radius from in-vivo high-resolution CT imaging. J Biomech.1999;32:821-8.

13 Bozic KJ, Keyak JH, Skinner HB, Bueff HU, Bradford DS. Three-dimensional finite element modeling of a cervical vertebra: an investigation of burst fracture mechanism. J Spine Disorders.1994;7(2):102-110.

14 Kim S, Lee SK. Snowboard wrist guards- use, efficacy, and design. A systematic review. Bulletin of the NYU Hospital for Joint Diseases. 2011;69(2):149-57.

15 Greenwald R, Janes P, Swanson S, McDonald T. Dynamic impact response of human cadaveric forearms using a wrist brace. Am J Sports Med.1998;26(6):825-830.

16 Hwang I, Kim K, Kaufman K et al. Biomechanical efficiency of wrist guards as a shock isolator.J Biomech Eng.2006;128(2):229-234.

17 Staebler M, Moore D, Akelman E et al. The effect of wrist guards on bone strain in the distal forearm. Am J Sports Med.1999;27(4):500-506.

18 Cheng S, Rajaratnam K, Raskin K et al. "Splint-top" fracture of the forearm: a description of an in-line skating injury associated with the use of protective wrist splints. $J$

Trauma.1995;39(6):1194-1197.

19 Snelderwaard PC, DeGroot JH, Deban SM. Digital video combined with conventional radiography creates an excellent high-speed x-ray video system. $J$

Biomechanics.2002;35:1007-1009.

${ }^{20}$ Mishima K, Hibiki T, Nishihara H. Visualization and measurement of two-phase flow by using neutron radiography. Nuclear Engineering and Design.1997;43:25-35.

${ }^{21}$ Naemura K, Ohta Y, Fujimoto T et al. Comparison of closing dynamics of mechanical prosthetic heart valves. American Society for Artificial Internal Organs (ASAIO).1997;43:401-404.

22 Stiehl JB, Komistek RD, Dennis DS, Paxson RD. Fluoroscopic analysis of kinematics after posterior cruciate retaining knee arthroplasty. J Bone Joint Surg (Br).1995;77-B:884-889.

${ }^{23} \mathrm{Li}$ G, Van de Velde SK, Bingham JT. Validation of a non-invasive fluoroscopic imaging technique for the measurement of dynamic knee joint motion. $J$

Biomechanics.2008;41:1616-1622.

\section{Figure Legend}

Figure 1: Schematic representation of drop tower assembly, using potted cadaver forearm

Figure 2: Schematic representation of recording system assembled around drop tower assembly, including xray source, image intensifier, video camera for xray images, and video camera for visible light images

Figure 3: Photographs of three wrist guard designs. From left: Seirus Jam Master II (3a and 3b), Dakine wrist guard (3c), and prototype design (3d)

Figure 4: Sequential frames of high-speed video xray of impact onto potted cadaver wrist, protected with Dakine volar wrist guard

Figure 5: Sequential frames of high-speed video impact onto potted cadaver wrist, protected with Dakine volar wrist guard 
